\title{
Interaction-driven breakdown of dynamical localization in a kicked quantum gas
}

\author{
Alec Cao \\ University of California, Santa Barbara https://orcid.org/0000-0002-6111-1375 \\ Roshan Sajjad ( $\nabla$ rsajjad@ucsb.edu ) \\ University of California, Santa Barbara https://orcid.org/0000-0002-1090-4092 \\ Hector Mas \\ University of California, Santa Barbara \\ Ethan Simmons \\ University of California, Santa Barbara \\ Jeremy Tanlimco \\ University of California, Santa Barbara https://orcid.org/0000-0002-7092-3316 \\ Eber Nolasco-Martinez \\ University of California, Santa Barbara \\ Toshihiko Shimasaki \\ UC Santa Barbara \\ Hasan Kondakci \\ University of California, Santa Barbara https://orcid.org/0000-0002-2567-5587 \\ Victor Galitski \\ University of Maryland \\ David Weld
}

University of California, Santa Barbara https://orcid.org/0000-0002-4574-9491

\section{Letter}

Keywords: quantum interference, dynamical localization, quantum chaos

Posted Date: October 25th, 2021

DOl: https://doi.org/10.21203/rs.3.rs-958231/v1

License: (1) This work is licensed under a Creative Commons Attribution 4.0 International License.

Read Full License 
Version of Record: A version of this preprint was published at Nature Physics on September 26th, 2022. See the published version at https://doi.org/10.1038/s41567-022-01724-7. 


\title{
Interaction-driven breakdown of dynamical localization in a kicked quantum gas
}

\author{
Alec Cao, ${ }^{1}$ Roshan Sajjad,${ }^{1}$ Hector Mas,${ }^{1}$ Ethan Q. Simmons,${ }^{1}$ Jeremy L. Tanlimco, ${ }^{1}$ Eber \\ Nolasco-Martinez, ${ }^{1}$ Toshihiko Shimasaki, ${ }^{1}$ H. Esat Kondakci, ${ }^{1}$ Victor Galitski, ${ }^{2}$ and David M. Weld ${ }^{1}$ \\ ${ }^{1}$ Department of Physics, University of California, Santa Barbara, California 93106, USA \\ ${ }^{2}$ Joint Quantum Institute and Department of Physics, \\ University of Maryland, College Park, MD 20742, USA
}

Quantum interference can terminate energy growth in a continually kicked system, via a single-particle ergodicity-breaking mechanism known as dynamical localization. The effect of many-body interactions on dynamically localized states, while important to a fundamental understanding of quantum decoherence, has remained unexplored despite a quarter-century of experimental studies. We report the experimental realization of a tunably-interacting kicked quantum rotor ensemble using a Bose-Einstein condensate in a pulsed optical lattice. We observe signatures of a prethermal localized plateau, followed for interacting samples by interaction-induced anomalous diffusion with an exponent near one half. Echo-type time reversal experiments establish the role of interactions in destroying reversibility. These results quantitatively elucidate the dynamical transition to many-body quantum chaos, advance our understanding of quantum anomalous diffusion, and delimit some possibilities for protecting quantum information in interacting driven systems.

Ergodicity breaking in quantum matter and relaxation dynamics of thermalizing phases are two aspects of a central question of non-equilibrium many-body physics: how and when do isolated quantum systems thermalize? A growing body of theoretical and experimental work suggests that ergodicity can be avoided or hindered by a variety of mechanisms, including many-body localization [1, 2], quantum many-body scarring [3, 4], and prethermalization [5-9]. Even without ergodicity break- 56 ing, the expected emergence of quantum chaos upon the 57 addition of interactions to driven systems is not well un- 58 derstood. For example, one ubiquitous but incompletely- 59 understood feature of the interface between localized and 60 ergodic regimes is anomalous diffusion $[10,11]$, which can 61 potentially serve as an indicator of entanglement spread- 62 ing [12]. A general predictive understanding of such phe- 63 nomena remains an open challenge to theory and exper- 64 iment.

The quantum kicked rotor (QKR) [15-17] is a paradig- 66 matic model of dynamical ergodicity breaking. While 67 strong, repeated kicking drives a classical rotor into 68 chaotic diffusion, the corresponding quantum rotor stops 69 absorbing energy after a finite time, signaling the on- 70 set of dynamical localization. Despite the complete ab- 71 sence of disorder, this phenomenon can be understood as 72 a manifestation of Anderson localization in momentum 73

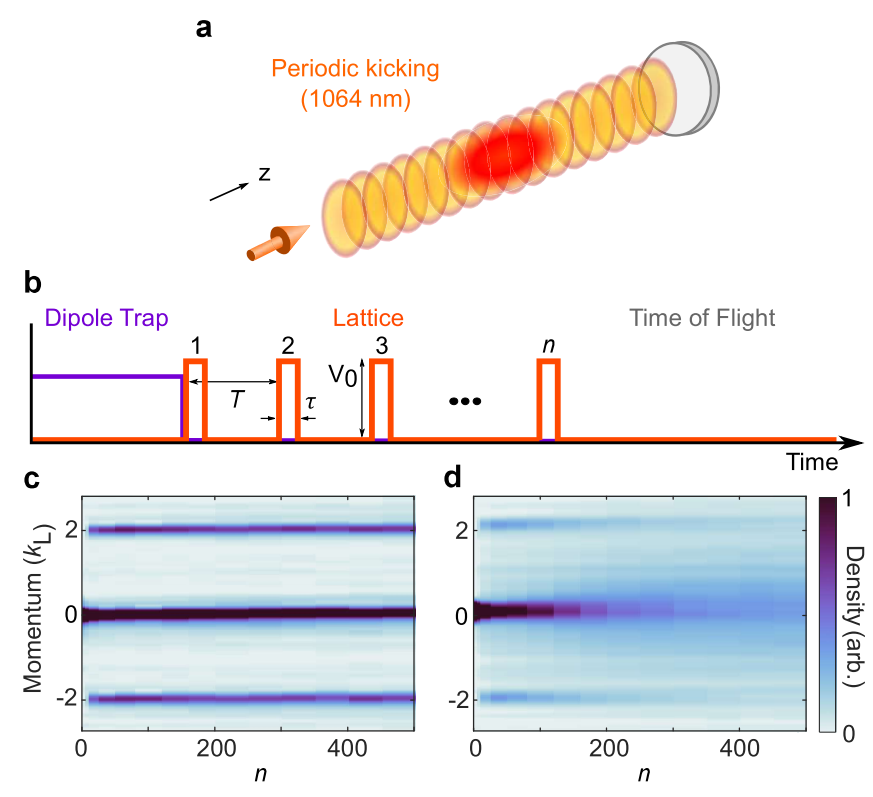

Fig. 1. Experimentally realizing an interacting quantum kicked rotor. (a) Schematic of BEC in single pulsed optical lattice. (b) Experimental sequence. After setting the scattering length the trap is removed and kicking is applied with period $T$, pulse width $\tau$, and amplitude $V_{0}$ for $n$ cycles. The atoms are imaged after a time-of-flight expansion [13]. (c-d) Measured axial momentum distribution versus kick number $n$ for noninteracting (c) and interacting (d) samples, revealing collisional momentum redistribution.

space $[18,19]$. Although the QKR is a natural context for experimental probes of the interplay between manybody interactions and dynamical localization, the quantum thermodynamics of interacting kicked rotors have not previously been experimentally explored. Depending on how interactions are introduced into the model, theoretical studies have predicted a variety of novel dynamical phenomena ranging from anomalous diffusion [20-23] to classical prethermalization [24] to many-body dynamical localization [25, 26].

Here we report the first experimental study of dynamical localization in the presence of tunable contact interactions, which are nonlocal in momentum space. These experiments investigate a ${ }^{7} \mathrm{Li}$ Bose-Einstein condensate (BEC) kicked $n$ times at period $T$ by a far-detuned optical lattice of spacing $d=532 \mathrm{~nm}$ and depth $V_{0}$ for duration $\tau$ (see Fig. 1). We report momentum and energy in units of $k_{\mathrm{L}}=\pi / d$ and $E_{\mathrm{R}}=\hbar^{2} k_{\mathrm{L}}^{2} / 2 m$ with $m$ the 

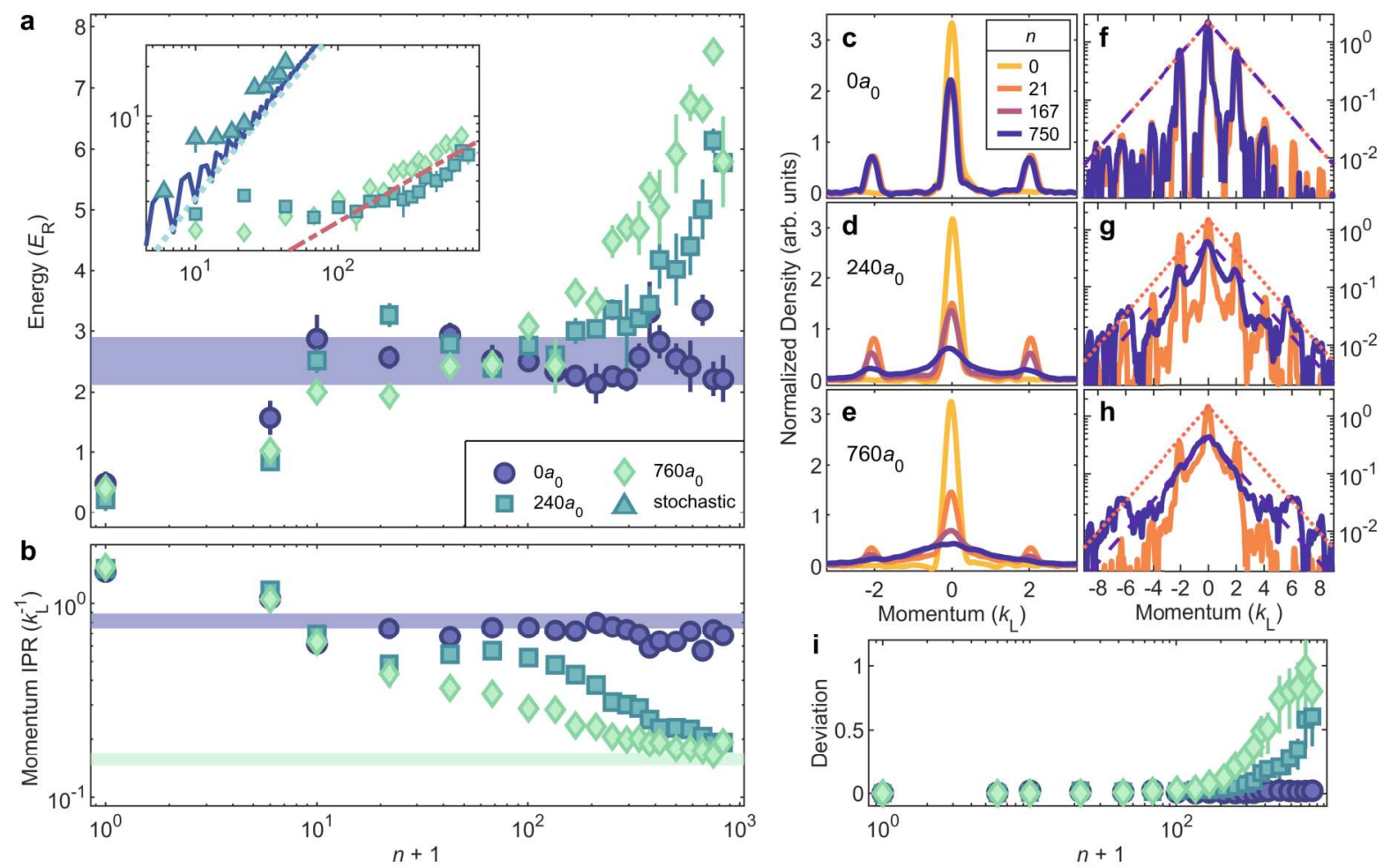

Fig. 2. Observing the interaction-induced emergence of quantum chaos. (a) Energy versus kick number for varying a. Blue horizontal shaded region indicates the measured single-rotor localization energy of $E_{\text {loc }}=2.5(4) E_{\mathrm{R}}$. Here $V_{0}=64 E_{\mathrm{R}}$, $T=1.2 \mu \mathrm{s}$ and $\tau=300 \mathrm{~ns}(K \approx 2.3$ and $k \approx 1.5)$. The inset contrasts interaction-induced delocalization and anomalous diffusion with classical diffusion caused by random kicking, the latter achieved by adding random offsets to the average kick spacing $T$ drawn uniformly from the interval $[-T / 4, T / 4]$. The solid curve is noninteracting quantum theory and the dotted line is a diffusion curve $4 D n / k^{2}$ with $D \approx 0.19$ extracted from the classical standard map [14]. The red dot-dashed line is a subdiffusive $\sqrt{n}$ law serving as a guide to the eye. (b) Momentum-space IPR with transverse dimensions integrated out. The shaded regions are predictions for two exponentially localized distributions with $1 / e$ localization length $k_{\mathrm{loc}}=\sqrt{E_{\mathrm{loc}}} \approx 1.6(1) k_{\mathrm{L}}$ [13]. $(\mathbf{c}-\mathbf{e})$ Normalized smoothed momentum space densities at various $n$. (f-h) The same densities on a logarithmic scale. The orange dotted and purple dashed lines are exponentially localized curves $\exp \left(-k / k_{\text {loc }}\right)$ with amplitudes normalized to match the peak of the measured distributions at the given $n$. (i) Deviation from exponential localization over time based on integrated ratio between measured and exponential distributions with error bars computed from uncertainty in $k_{\text {loc }}[13]$.

mass of ${ }^{7} \mathrm{Li}$. The single-particle QKR is defined by the 1- 94 cycle Floquet map $U=e^{-i \hbar k^{2} / 2} e^{-i K \cos z / \hbar}$ describing a 95 sharp cosine potential impulse followed by free evolution. 96 Here $k$ and $z$ are momentum and position respectively, 97 $K=\hbar V_{0} \tau / 2 \hbar$ is the stochasticity parameter character- 98 izing kicking strength and $\hbar=8 E_{\mathrm{R}} T / \hbar$ is an effective 99 Planck's constant determined by the kick period. Ab-100 sorption imaging after free expansion is used to measure101 the momentum distribution; see the supplementary text102 for a full analysis of systematic effects in this procedure, 103 such as dynamics transverse to the lattice direction. In-104 teratomic interactions are varied by tuning the $s$-wave105 scattering length $a$ (reported in units of the Bohr radius ${ }^{100}$ $a_{0}$ ) using a magnetic Feshbach resonance. While the kick-107 ing primarily couples discrete momentum states along a ${ }^{108}$ single dimension, the atoms are entirely unconfined be-109 tween kicks; scattering between momentum modes thus110 couples the system to a bath of transverse free-particle ${ }^{111}$ states.

The main result of this work is presented in Fig. 2a. ${ }^{113}$
While a noninteracting sample exhibits dynamical localization, saturating to a finite energy for over 800 kicks, interacting samples clearly demonstrate the destruction of the dynamically localized plateau with increasing scattering length. At intermediate interaction strength $\left(a=240 a_{0}\right)$, we observe saturation to the same energy as non-interacting samples for approximately 300 kicks, suggesting the existence of a reasonably long-lived prethermal state. In contrast, the $760 a_{0}$ trace exceeds this localized energy after around 100 kicks; whether a quasiequilibrium dynamical state is truly established in this stronger-interacting sample is less clear. Fig. $2 \mathrm{~b}$ shows another aspect of the same evolution, plotting the momentum space inverse participation ratio (IPR) versus kick number. The IPR characterizes the number of states over which the system is distributed, thereby also probing how collisional momentum redistribution washes out the originally discrete momentum modes, a process less easily inferred from energy measurements. While the $240 a_{0}$ data exhibit a clear steady-state behavior for 100 
kicks, the $760 a_{0}$ IPR decreases monotonically for almost the entire experiment.

A second key result of these measurements is that the observed delocalizing dynamics clearly exhibit anomalous diffusion: it appears that even interacting quantum kicked rotors absorb energy much more slowly than classical rotors. The inset of Fig. 2a compares the nature of the observed interaction-induced subdiffusive delocalization with linear energy growth in the classically chaotic model. We experimentally simulate classical dynamics by adding stochastic fluctuations to the kicking period $T$, making use of the known sensitivity of dynamical localization to timing noise [27]. These experimental data agree both with single-particle quantum numerics and with the linear energy growth predicted by the classical standard map [14], and stand in clear contrast to the measured interaction-induced anomalous diffusion away from the dynamically localized state. The dot-dashed red line indicates a $\sqrt{n}$ energy growth, and fitting the latetime data to $n^{\alpha}$ yields anomalous diffusion exponents $\alpha$ in the range $[0.4,0.6]$. For reference, $1 \mathrm{D}$ Gross-Pitaevskii simulations on a ring [22] predict $\alpha \in[0.5,0.8]$, though a direct quantitative comparison to theory is challenging due to the significant condensate depletion and the three-dimensional nature of the experiment. Theoretical studies of the effect of local nonlinearity on real-space Anderson localization instead suggest $\alpha \in[0.3,0.4]$ [28, 29], but the long-range nature of contact interactions in momentum space similarly complicates comparison. This clear observation of anomalous diffusion in the interacting quantum kicked rotor raises a variety of fascinating questions for future exploration. What correlations are responsible for the anomalous diffusion dynamics? What feature of the interacting system prevents the interacting ${ }_{172}$ QKR from heating classically? What theoretical frame- ${ }^{-173}$ work is appropriate for quantitatively predicting wave- ${ }_{174}$ function evolution in this regime? What are the univer- ${ }_{175}$ sality properties of the subdiffusive exponent?

For further insight into the dynamics of kicked inter-177 acting quantum systems we examine the evolution of the ${ }^{178}$ momentum distribution, shown in Figs. 2c-e. We observe ${ }^{179}$ a clear distinction between the noninteracting samples, ${ }^{180}$ which settle at a sharply-peaked dynamically-localized ${ }_{181}$ momentum distribution, and the interacting samples,182 which gradually smear out in momentum space due to183 scattering. Plotting these same densities on a logarithmic ${ }_{184}$ scale in Fig. 2f-h illuminates the destruction of dynami-185 cal Anderson localization by assessing the departure from ${ }_{186}$ exponentially-localized Floquet states. The smeared-out 187 lower-energy modes actually appear to maintain the ex-188 pected localization length, and thus do not trivially in-189 dicate a departure from exponential localization. This ${ }_{190}$ observation is also reflected in the fact that two pre-191 dictions based on exponentially localized distribution ${ }_{192}$ bound the measured IPR in Fig. 2b. Instead, the de-193 parture from exponential localization manifests in the 194 emergence of increased relative population in the tails 195 of the distribution. It is interesting to note that recent 190
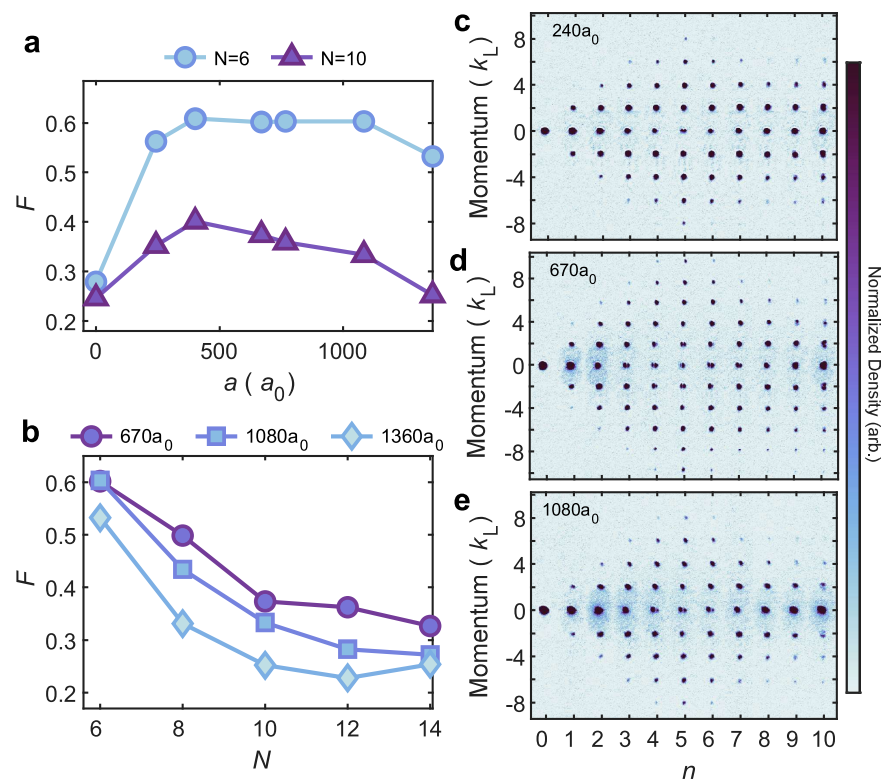

Fig. 3. Effect of interactions on reversibility in Loschmidt echo experiments. (a) Measured Loschmidt echo fidelity $F$ for a range of scattering lengths $a=[0,1500] a_{0}$ for $N=6$ (blue circles) and $N=10$ (purple triangles), where $N$ indicates the total number of kicks; a first set of $N / 2$ kicks propagates the system forward in time and a second timereversal set of $N / 2$ kicks propagates it backwards. (b) Measured fidelity $F$ at three different interaction strengths as a function of total number of kicks $N$ in a Loschmidt echo experiment. (c-e) Averaged absorption images of a BEC after the first $n$ kicks of an $N=10$ Loschmidt echo protocol, for three different $a$.

theory suggests that even many-body dynamically localized phases are expected to exhibit universal power-law decaying tails [30]. In Fig. 2i we quantitatively characterize the overall deviation from exponential localization [13], revealing a break time near 200 kicks for both interaction strengths. These findings provide a second experimental signature of the destruction of the dynamically localized state by interactions, now both at the level of macroscopic observables and squared wavefunctions.

The onset of energy delocalization due to interactions indicates a transition to the regime of many-body quantum chaos, which can be probed directly by studying time-reversal dynamics $[31,32]$. In Fig. 3 we probe the onset of chaotic dynamics by measuring the effect of interactions on a Loschmidt echo time-reversal protocol $[33,34]$. The echo is realized using quantum resonances [35] which occur for $k=2 \pi q$ with $q$ rational; in particular for $q=2(T \approx 9.95 \mu \mathrm{s})$, the free evolution in $U$ largely vanishes and effective time reversal can be achieved by setting $q$ to 1 for a single kick halfway through the sequence $[13,33,34]$. This procedure would create exact time reversal for a single zeroquasimomentum state in the absence of interactions. Due to finite quasimomentum spread and non-reversed interactions, the reversal is imperfect, yielding a Loschmidt 
fidelity $F=\left|\left\langle\psi\left|U_{2}^{\dagger} U_{1}\right| \psi\right\rangle\right|^{2}$ where $U_{1}$ and $U_{2}$ are time-229 evolution operators differing by some perturbation. Per- ${ }^{230}$ haps surprisingly, $F$ initially increases as the scattering length $a$ is turned up from zero. In this regime $U_{1} \operatorname{and}^{232}$ $U_{2}$ are primarily distinguished by the failure to reverse ${ }^{233}$ kinetic energy, and thus the increase can be explained by ${ }^{234}$ Thomas-Fermi reduction of the initial state momentum ${ }^{233}$ spread. Eventually, for large enough $a$, the interaction ${ }^{236}$ becomes the primary perturbation and $F$ begins to de- ${ }^{237}$ crease with $a$, marking the transition to predominantly ${ }_{239}^{238}$ interaction-induced irreversibility. The decay of fidelity with total number of kicks in a Loschmidt echo experiment is shown in Fig. 3b. The use of Loschmidt echo ${ }^{24}$ techniques as a probe of many-body quantum chaos not ${ }^{242}$ only illuminates the origins of the delocalizing dynam- ${ }^{243}$ ics we observe, but opens up the possibility of extending ${ }^{244}$ these protocols to probe scrambling in many-body quan- ${ }^{24}$ tum chaotic systems [36].

In conclusion, we have experimentally realized a many-249 body ensemble of quantum kicked rotors. Following the 250 evolution of interacting samples over hundreds of kicks,251 we observe signatures of an initial prethermal state, fol-252 lowed by an interaction-induced breakdown of dynam-253 ical localization via anomalous diffusion, signaling the 254 onset of many-body quantum chaos. Characterization 255 of the departure from the dynamically localized state 256 indicates subdiffusive energy growth with an exponent 257 near 0.5 , easily distinguishable from classical Joule heat-258 ing in a randomly kicked system, and reveals momentum 259 space distributions which are not exponentially local-260 ized. Measuring Loschmidt echo time-reversal dynamics261 with a quantum resonance enabled us to directly probe262 the role of interaction-induced irreversibility in driving a transition to many-body quantum chaos. Together, these results demonstrate and quantitatively illuminate the emergence of interaction-driven quantum chaos in a paradigmatic localized system.

We acknowledge helpful conversations with Adam Rançon, Norman Yao, and Thomas Schuster. During the performance of the research described here we became aware of related efforts underway in another experimental group [37] which have reached similar conclusions using a complementary approach. Funding: D.M.W. acknowledges support from the Air Force Office of Scientific Research (AFOSR FA9550-20-1-0240), the Army Research Office (PECASE W911NF1410154), and the National Science Foundation (CAREER 1555313 and QLCI OMA-2016245). D.M.W., R.S., and E.NM. acknowledge support from the UCSB NSF Quantum Foundry through the Q-AMASE-i program (Grant No. DMR-1906325). V.G. was supported by US-ARO Contract No.W911NF1310172, NSF DMR-2037158, and the Simons Foundation. Authors' contributions: A.C., R.S., H.M., E.Q.S., J.L.T., E.N-M., T.S. and H.E.K. contributed to operating the experiment and performing the measurements. A.C., R.S. and H.M. analyzed the data. A.C. conceptualized and performed the theoretical simulations of rotor and spin models. V.G. and D.M.W. developed the idea for the experiment. D.M.W. supervised the work. A.C., R.S., H.M., V.G. and D.M.W. wrote the manuscript. All authors contributed to discussion and interpretation of the results. Competing interests: The authors declare no competing interests. Data and materials availability: All data needed to evaluate the conclusions in this study are presented in the paper and in the supplementary materials.
[1] R. Nandkishore and D. A. Huse, Annu. Rev. Condens.285 Matter Phys. 6, 15 (2015).

[2] D. A. Abanin, E. Altman, I. Bloch, and M. Serbyn, Rev.287 Mod. Phys. 91, 021001 (2019).

[3] H. Bernien, S. Schwartz, A. Keesling, H. Levine, A. Om-28 ran, H. Pichler, S. Choi, A. S. Zibrov, M. Endres, 290 M. Greiner, V. Vuletić, and M. D. Lukin, Nature 551,291 579 (2017).

[4] M. Serbyn, D. A. Abanin, and Z. Papić, Nature Physics293 17, 675 (2021).

[5] K. Singh, C. J. Fujiwara, Z. A. Geiger, E. Q. Sim-295 mons, M. Lipatov, A. Cao, P. Dotti, S. V. Rajagopal,296 R. Senaratne, T. Shimasaki, M. Heyl, A. Eckardt, and 297 D. M. Weld, Phys. Rev. X 9, 041021 (2019).

[6] A. Rubio-Abadal, M. Ippoliti, S. Hollerith, D. Wei,299 J. Rui, S. L. Sondhi, V. Khemani, C. Gross, and I. Bloch, 300 Phys. Rev. X 10, 021044 (2020).

[7] P. Peng, C. Yin, X. Huang, C. Ramanathan, and P. Cap-302 pellaro, Nat. Phys. (2021).

[8] T. Langen, S. Erne, R. Geiger, B. Rauer, T. Schweigler,304 M. Kuhnert, W. Rohringer, I. E. Mazets, T. Gasenzer,305 and J. Schmiedmayer, Science 348, 207 (2015).
[9] M. Ueda, Nature Reviews Physics 2, 669 (2020).

[10] K. Agarwal, S. Gopalakrishnan, M. Knap, M. Müller, and E. Demler, Phys. Rev. Lett. 114, 160401 (2015).

[11] S. A. Weidinger and M. Knap, Sci. Rep. 7, 1 (2017).

[12] R. Menu and T. Roscilde, Phys. Rev. Lett. 124, 130604 (2020).

[13] Materials and methods are available as supplementary materials.

[14] B. V. Chirikov, Phys. Rep. 52, 263 (1979).

[15] F. L. Moore, J. C. Robinson, C. F. Bharucha, B. Sundaram, and M. G. Raizen, Phys. Rev. Lett. 75, 4598 (1995).

[16] J. Chabé, G. Lemarié, B. Grémaud, D. Delande, P. Szriftgiser, and J. C. Garreau, Phys. Rev. Lett. 101, 255702 (2008).

[17] B. Gadway, J. Reeves, L. Krinner, and D. Schneble, Phys. Rev. Lett. 110, 190401 (2013).

[18] S. Fishman, D. R. Grempel, and R. E. Prange, Phys. Rev. Lett. 49, 509 (1982).

[19] D. R. Grempel, R. E. Prange, and S. Fishman, Phys. Rev. A 29, 1639 (1984).

[20] D. L. Shepelyansky, Phys. Rev. Lett. 70, 1787 (1993). 
21] G. Gligorić, J. D. Bodyfelt, and S. Flach, Europhys. Lett.371 96, 30004 (2011).

[22] S. Lellouch, A. Rançon, S. De Bièvre, D. Delande, and 373 J. C. Garreau, Phys. Rev. A 101, 043624 (2020)

[23] A. Russomanno, M. Fava, and R. Fazio, Phys. Rev. B 375 103, 224301 (2021).

24] A. Rajak, I. Dana, and E. G. Dalla Torre, Phys. Rev. B 377 100, 100302 (2019).

[25] C. Rylands, E. B. Rozenbaum, V. Galitski, and R. Konik, 379 Phys. Rev. Lett. 124, 155302 (2020).

[26] A. C. Keser, S. Ganeshan, G. Refael, and V. Galitski, 381 Phys. Rev. B 94, 085120 (2016).

[27] W. H. Oskay, D. A. Steck, and M. G. Raizen, Chaos, Solitons and Fractals 16, 409 (2003).

[28] A. S. Pikovsky and D. L. Shepelyansky, Phys. Rev. Lett. 100, 094101 (2008).

[29] S. Flach, D. O. Krimer, and C. Skokos, Phys. Rev. Lett. 102, 024101 (2009).

[30] V. Vuatelet and A. Rançon, Effective thermalization of a many-body dynamically localized bose gas (2021), arXiv:2103.14388 [cond-mat.quant-gas].

[31] E. B. Rozenbaum, S. Ganeshan, and V. Galitski, Phys. Rev. Lett. 118, 086801 (2017).

[32] T. Gorin, T. Prosen, T. H. Seligman, and M. Žnidarič, Phys. Rep. 435, 33 (2006).

[33] A. Ullah and M. D. Hoogerland, Phys. Rev. E 83, 046218 (2011).

[34] J. Martin, B. Georgeot, and D. L. Shepelyansky, Phys. Rev. Lett. 100, 044106 (2008).

[35] M. Lepers, V. Zehnlé, and J. C. Garreau, Phys. Rev. A 77, 043628 (2008).

[36] B. Swingle, G. Bentsen, M. Schleier-Smith, and P. Hayden, Phys. Rev. A 94, 040302 (2016).

[37] J. H. S. Toh, K. C. McCormick, X. Tang, Y. Su, X.-W. Luo, C. Zhang, and S. Gupta, Observation of many-body dynamical delocalization in a kicked ultracold gas (2021), arXiv:2106.13773 [cond-mat.quant-gas].

[38] S. E. Pollack, D. Dries, M. Junker, Y. P. Chen, T. A. Corcovilos, and R. G. Hulet, Phys. Rev. Lett. 102, 090402 (2009).

[39] P. Deuar and P. D. Drummond, Phys. Rev. Lett. 98, 120402 (2007).

[40] A. Tenart, C. Carcy, H. Cayla, T. Bourdel, M. Mancini, and D. Clément, Phys. Rev. Res. 2, 013017 (2020).

[41] B. Gadway, D. Pertot, R. Reimann, M. G. Cohen, and D. Schneble, Opt. Express 17, 19173 (2009).

[42] Y. B. Ovchinnikov, J. H. Müller, M. R. Doery, E. J. D. Vredenbregt, K. Helmerson, S. L. Rolston, and W. D. Phillips, Phys. Rev. Lett. 83, 284 (1999).

[43] B. Klappauf, W. Oskay, D. Steck, and M. Raizen, Physica D 131, 78 (1999).

[44] S. Fishman, I. Guarneri, and L. Rebuzzini, J. Stat. Phys. 110, 911 (2003).

[45] I. Dana and D. L. Dorofeev, Phys. Rev. E 73, 026206 (2006).

[46] L. D'Alessio and M. Rigol, Phys. Rev. X 4, 041048 (2014).

[47] T. Boness, S. Bose, and T. S. Monteiro, Phys. Rev. Lett. 96, 187201 (2006).

[48] T. Boness, K. Kudo, and T. S. Monteiro, Phys. Rev. E 81, 046201 (2010).

[49] M. Rigol, V. Dunjko, and M. Olshanii, Nature 452, 854 (2008).

[50] C. Monroe, W. C. Campbell, L.-M. Duan, Z.-X. Gong,
A. V. Gorshkov, P. W. Hess, R. Islam, K. Kim, N. M. Linke, G. Pagano, P. Richerme, C. Senko, and N. Y. Yao, Rev. Mod. Phys. 93, 025001 (2021).

[51] A. Browaeys and T. Lahaye, Nat. Phys. 16, 132 (2020).

[52] Y. Salathé, M. Mondal, M. Oppliger, J. Heinsoo, P. Kurpiers, A. Potočnik, A. Mezzacapo, U. Las Heras, L. Lamata, E. Solano, S. Filipp, and A. Wallraff, Phys. Rev. X 5, 021027 (2015).

[53] L. M. Sieberer, T. Olsacher, A. Elben, M. Heyl, P. Hauke, F. Haake, and P. Zoller, npj Quantum Inf. 5, 1 (2019).

[54] N. Goldman and J. Dalibard, Phys. Rev. X 4, 031027 (2014). 


\section{Methods}

Experimental platform and sequence. The experi-43. ments begin with a Bose-Einstein condensate (BEC) of 432 around $10^{5}{ }^{7} \mathrm{Li}$ atoms in a far-detuned optical dipole ${ }_{43}$ trap with trapping frequencies $\omega_{x, z} / 2 \pi \approx 40 \mathrm{~Hz}$ and434 $\omega_{y} / 2 \pi \approx 56 \mathrm{~Hz}$, where $z$ is the axis of the optical lat-435 tice, $y$ is the direction of gravity, and $x$ is the remaining ${ }_{436}$ orthogonal axis. The condensate is produced by optical ${ }_{437}$ evaporation at an $s$-wave scattering length of $a=240 a_{0,438}$ set by an applied magnetic field in the vicinity of the 439 broad Feshbach resonance at 737 Gauss [38]. Immedi-440 ately after evaporation, the fields are ramped to their desired value over $60-90 \mathrm{~ms}$ and maintained for the re-441 mainder of the experiment. The dipole trap is then ex-442 tinguished and the BEC repeatedly subjected to a pulsed ${ }^{443}$ $1 \mathrm{D}$ optical lattice with lattice constant $d=532 \mathrm{~nm}$, laser444 wave vector $k_{\mathrm{L}}=\pi / d$, and recoil energy $E_{\mathrm{R}}=\hbar^{2} k_{\mathrm{L}}^{2} / 2 m^{445}$ with $m$ the mass of ${ }^{7} \mathrm{Li}$. The full dynamics are then well 446 described by the second-quantized Hamiltonian

$$
\begin{gathered}
\mathcal{H}=\int \mathrm{d}^{3} r \hat{\Psi}^{\dagger}(\mathbf{r}, t) H(\mathbf{r}, t) \hat{\Psi}(\mathbf{r}, t)+ \\
\frac{g}{2} \int \mathrm{d}^{3} r \hat{\Psi}^{\dagger}(\mathbf{r}, t) \hat{\Psi}^{\dagger}(\mathbf{r}, t) \hat{\Psi}(\mathbf{r}, t) \hat{\Psi}(\mathbf{r}, t) \\
H(\mathbf{r}, t)=\frac{p^{2}}{2 m}+\frac{V_{0}}{2} \cos \left(2 k_{L} z\right) I(x, y) \sum_{n} f_{\tau}(t-n T) .
\end{gathered}
$$

The key kick parameters are the lattice depth $V_{0}$, ef- ${ }^{450}$ fective pulse width $\tau$, and kick period $T$. $V_{0}$ is calibrated ${ }_{461}^{460}$ through a standard Kapitza-Dirac diffraction technique. ${ }_{462}^{461}$ $f_{\tau}(t)$ denotes a unit amplitude pulse function beginning ${ }_{463}^{462}$ at $t=0$ of width $\tau$. The experimental pulse is approxi- ${ }^{463}$ mated by a piecewise function with a linear rise and fall ${ }^{464}$ of $200 \mathrm{~ns}$ duration before and after a plateau of variable hold time. For the experimental data in the main text ${ }^{466}$ with $\tau=300 \mathrm{~ns}$ between the half-maximum points, this ${ }^{467}$ hold duration is $100 \mathrm{~ns}$. The scattering length $a$ deter- ${ }^{468}$ mines the two-body coupling coefficient $g=4 \pi \hbar^{2} a / \mathrm{m}^{469}$ Here $I(x, y)$ denotes the transverse intensity profile of the lattice beams normalized to unity maximum; this is ${ }_{472}^{471}$ approximately Gaussian $I(x, y) \approx e^{-2\left(x^{2}+y^{2}\right) / \sigma^{2}}$ with $\mathrm{a}_{473}^{47}$ measured $1 / e^{2}$ beam radius of $\sigma \approx 65 \mu \mathrm{m}$. The total $\mathrm{l}_{474}$ duration of kicking is at most $1 \mathrm{~ms}$ for our longest exper- ${ }^{475}$ iments, significantly shorter than the $4 \mathrm{~ms}$ it takes the $\mathrm{A}_{476}$ BEC to fall under the influence of gravity through the lattice beam waist.

To measure the momentum distribution, we perform ${ }_{479}^{478}$ absorption imaging of the atoms after free expansion. ${ }_{480}$ The time-of-flight (TOF) duration is $3.5 \mathrm{~ms}$ for the $\mathrm{de}_{-481}{ }^{480}$ localization data and $2 \mathrm{~ms}$ for the Loschmidt data. Due to the low mass of ${ }^{7} \mathrm{Li}$ and the breadth of the Feshbach ${ }_{483}^{482}$ resonance, coil inductance prevents sweeping the mag- ${ }_{484}$ netic fields to the noninteracting regime for this expan- ${ }_{485}$ sion period. This means additional scattering occurs dur- ${ }^{486}$ ing expansion, which may lead to systematic errors in the measured quantities (see supplementary section 6). For the energy, we are able to account for this scattering in our analysis due to the energy-conserving nature of the collisions. For metrics such as the IPR, this systematic is challenging to avoid. However by tracking the evolution of these observables as a function of kick number at a fixed TOF duration, we can largely attribute the qualitative observed dynamics to the evolution under the Hamiltonian (1) as opposed to the expansion. At large $n$, the majority of scattering happens during the kicking duration so expansion effects become negligible.

Delocalization data analysis. This section discusses the analysis behind Fig. 2. Because the momentum distributions of the interacting samples change significantly over the course of the delocalization experiments, the quantities shown in Fig. 2 are computed directly from raw or averaged images as opposed to fitting procedures. However, this can make measurable quantities such as energy sensitive to noise, especially near the edge of the camera sensor due to the quadratic weighting. To maximize the signal-to-noise ratio in our measurement, we analyze raw images using an adaptive region-of-interest (ROI). First, a single base ROI capturing all detectable atoms at all times is created for each interaction strength. The integrated density in this ROI is used to post-select images with total atom numbers falling within a $\pm 10 \%$ window of the mean, in order to reduce variations in the interaction energy, which depends directly on atom density. For these data we take 10 images at each kick number, of which typically 4-7 are discarded by this postselection procedure. The ROI boundaries at each kick number are then determined by the points at which the cumulative summed distributions of the averaged image outward from a center point reach a threshold value. The thresholds are set empirically and the boundaries obtained by the following procedure. First we compute the transverse bound by integrating out the entire axial direction to get the overall transverse distribution, find the point it crosses an $85 \%$ threshold and then expand the resulting boundary by a factor of 1.5 (1.2 in the delocalization data of supplementary section 1) to ensure all atoms are captured. We then compute an axial boundary going point by point along the transverse direction; at each transverse point we integrate over 10 neighboring transverse pixel rows to get a "local" axial distribution, find the point it crosses a $99.8 \%$ threshold and expand by a factor 1.15. Finally we smooth each ROI boundary and take a moving average across different kick numbers ( 4 on each side). Crucially, we have confirmed that the qualitative observation of delocalization is not significantly altered from the simple case where we use just the initial single base ROI across all shots. However, the details of the trends should be more accurately captured by the adaptive procedure because the signal-to-noise ratio over the ROI is optimized at each kick number. All measurable quantities are then calculated from the imaged densities within this region. 
Since we do not observe any substantial atom loss dur-545 ing the kicking duration, we treat the imaged atomic 546 densities as normalized distributions. For Figs. 2a-b, we 547 compute the measured quantities from individual exper-548 imental runs and then average the results, with the re-549 ported error bar as the standard error of the mean. For 550 Fig. 2i, we instead compute the averaged distributions551 first before computing the deviation from exponential 552 localization; the errorbars are computed from a Monte-553 Carlo simulation of the uncertainty in $k_{\text {loc }}$ discussed later 554 in this section. A smoothing filter is applied to the dis-555 played densities in Figs. 2c-h for visual clarity, but not in 556 the subsequent calculation of the localization deviation 557 in Fig. 2i.

To measure the energy, we compute the post-expansion559 spatial variance of the distribution in both the kicking $z 560$ and transverse $x$ directions of the image. Assuming cylin-561 drical symmetry, the kinetic energy is then calculated as562 $m\left(\left\langle z^{2}\right\rangle+2\left\langle x^{2}\right\rangle\right) / 2 t_{\text {TOF }}^{2}$ with $t_{\text {TOF }} \approx 3.5 \mathrm{~ms}$ (see section563 2.4 for a discussion of possible corrections to the conver-564 sion of position to energy). For an accurate measurement ${ }_{565}$ of the interacting samples, inclusion of the transverse energy is necessary to account for energy-conserving scat- ${ }^{566}$ tering processes that occur both during the kicking and ${ }^{567}$ TOF. In addition, the inhomogeneous intensity profile of ${ }^{568}$ the beam $I(x, y)$ leads to a transverse energy oscillation ${ }^{569}$ in all samples including the noninteracting ones (see sup-570 plementary section 5). Since we are not interested in this ${ }^{571}$ effect, we remove it to leading order by subtracting off ${ }^{572}$ the noninteracting transverse energy from each trace, $\mathrm{so}^{573}$ that the noninteracting energy is purely the kinetic en- ${ }^{574}$ ergy along the kicking direction. To compute the error ${ }^{575}$ bars on the interacting data, we add the error of the to-576 tal interacting energy and noninteracting transverse en-577 ergy in quadrature. The single-particle localization en-578 ergy $E_{\text {loc }}$ is estimated by averaging the noninteracting ${ }^{579}$ trace for $n \geq 100$, and the reported uncertainty is based ${ }^{580}$ on the standard deviation of those points. We note that ${ }^{581}$ this uncertainty is not only due to experimental imper-582 fections, but also due to natural dynamical fluctuations, ${ }^{583}$ as evidenced by the results of noninteracting simulations ${ }^{584}$ like those shown in Fig. S3.

We compute an effective 1D momentum-space IPR by ${ }^{586}$ first integrating out the transverse dimension and then ${ }^{57}$ summing the squares of the subsequent normalized $\mathrm{ax}^{-588}$ ial density. We confirmed that this qualitatively matches ${ }^{589}$ the result of directly integrating the squared $2 \mathrm{D}$ distri- ${ }^{590}$ bution while largely eliminating the beam-induced trans- ${ }^{591}$ verse oscillation. Specifically for computing this met-- ${ }^{592}$ ric, we apply a smoothing filter to the normalized densi- ${ }^{593}$ ties consistently across all 3 interaction strengths. This ${ }^{594}$ suppresses high-frequency background noise which sets a lower bound on the measurable IPR due to the squaring ${ }^{595}$ procedure. The measured values are compared to two ${ }^{590}$ predictions based on an exponentially localized distribu- ${ }^{597}$ tion. The blue shaded region is obtained by numerically ${ }^{598}$ computing the IPR for the momentum space distribution ${ }^{599}$ $\exp \left(-|k| / k_{\text {loc }}\right) \sum_{j} \exp \left(-\left(k-2 k_{\mathrm{L}} j\right)^{2} / w^{2}\right)$, which models ${ }^{600}$ a Gaussian comb with an exponential envelope. This is a reasonable expectation for a finite-size, localized noninteracting condensate occupying only discrete momentum modes. The width parameter $w$ is measured from fitting the $n=0$ noninteracting condensate and takes into account the momentum-space resolution of the TOF given the finite condensate spatial extent. The width of the region is based on Monte Carlo simulation of uncertainty in $k_{\text {loc }}$, where the resulting distribution is fit to a Gaussian to extract the mean and standard deviation. The green shaded region is calculated analytically for a pure exponential distribution of infinite extent and is given by $1 / 4 k_{\text {loc }}$. Taking into account the finite width of the imaging region changes the distribution normalization and leads to the following correction factor $\left(1-\exp \left(-2 k_{0} / k_{\text {loc }}\right) /\left(1-\exp \left(-k_{0} / k_{\text {loc }}\right)\right)^{2}\right.$; here $k_{0} \approx 9.85 k_{L}$ is the half-width of our images which yields a negligible correction factor of $\approx 1.006$. The width of the region is computed through linearized error propagation.

In Fig. 2i, the plotted localization metric is $\int_{-k_{0}}^{k_{0}} \max [r(k)-1,0] \mathrm{d} k / 2 k_{0}$. Here, $r(k)=$ $|\psi(k)|^{2} / \exp \left(-k / k_{\text {loc }}\right)$ is the ratio of the measured axial density denoted $|\psi(k)|^{2}$ and an exponential localization envelope. Here the maximum of $|\psi(k)|^{2}$ is set to unity. Taking the maximum of $r(k)-1$ and 0 ensures that the result is only sensitive to regions of the distribution which decay more slowly than exponentially. That is, it interprets 0 as "at least exponentially" localized with respect to a given localization length, and thus characterizes departures from a given dynamically localized state in the traditional sense of exponentially localized wavefunctions. We note however that the system remaining exponentially localized but with a larger localization length would result in a non-zero value for this metric, which motivates the direct inspection of the distributions in Figs. 2f-h. The reported values and errorbars are extracted by propagating a Gaussian uncertainty in the measured $k_{\text {loc }}$ through a Monte-Carlo simulation. We find that the resulting distributions interpolate between sharply peaked at 0 with a rapid fall-off when well-localized, to positively skewed with non-zero peak in the delocalized regime. We empirically find that a log-normal distribution fits the Monte Carlo result well, and we use this fit to extract the data reported in Fig. 2i. In particular, the markers indicate the mean of the distribution and the errorbars represent the interquartile range containing the central $50 \%$ of the distribution. Because of the skewness, we investigate the Monte Carlo simulated distributions in more detail in supplementary section 2 .

Loschmidt experimental sequence and data analysis. Here we discuss the methods and analysis used to produce Fig. 3. The Loschmidt experiments begin similarly to the previously described sequence; for an $N$ kick Loschmidt sequence, the BEC is first kicked $N / 2$ times near quantum resonance at the parameters $V_{0} \approx 50 E_{\mathrm{R}}$, 
$\tau=300 \mathrm{~ns}$, and $T=9.93 \mu \mathrm{s}$. For this data, we adjusted 658 the lattice depth $V_{0}$ for different interaction strengths to achieve the same amount of absorbed energy after the first $N / 2$ kicks. This compensates for a decrease in energy absorption at the same lattice depth for higher interaction strengths, which we attribute to the increase of the Thomas-Fermi radius of the BEC relative to the lattice beam size. Neglecting this effect would artificially enhance the fidelity at very large interaction strengths due to a reduction in the effective stochasticity parameter $K$. We plot the zero mode fraction after the first $N / 2$ kicks without time-reversal (denoted $F^{\prime}$ Gauss) in Fig. S6 to benchmark this kicking amplitude normalization procedure.

After the first $N / 2$ kicks, we wait a half period $T / 2$ to shift the wavefunction spatially by half a lattice spacing, causing the sign of subsequent kicks to be reversed. We then apply another sequence of $N / 2$ kicks using the same lattice parameters to complete the echo sequence. The time series in Fig. 3c-e show absorption images averaged over 5 shots for each kick number $n$ in a $N=10$ experiment. Since we begin with a zero-momentum condensate mode, to measure the Loschmidt fidelity we simply need to count the fraction of atoms remaining in this mode. While atoms in other momentum modes coupled by the lattice are easily distinguished, atoms that have undergone scattering events into a smeared-out background distribution are not always well-separated. Thus, to extract the return fraction we fit the axial atomic distribution around the zero-momentum mode with a pair of Gaussians of varying width. The narrower Gaussian accounts for atoms remaining in the zero-momentum condensate after expansion, while the broader Gaussian measures the atoms that have been collisionally ejected from the condensate [39, 40]. In Fig. 3b, we show the fraction of atoms remaining in the narrow Gaussian and use this quantity as an estimate of the Loschmidt fidelity. Scattering during the expansion means that this necessarily underestimates the true fidelity, a possibility further addressed in supplementary section 6 .

Noninteracting QKR numerics. One-dimensional simulations of the noninteracting kicked rotor problem for comparison with experimental data are executed in two ways. We either perform a split-step Fourier integration of the QKR Hamiltonian (2) (ignoring the transverse distribution $I(x, y))$ to model the finite-width pulse shapes, or iterate the QKR Floquet map described in the main text. The simulations are typically performed with periodic boundary conditions over a single lattice site (except when modeling the TOF readout; see supplementary section 4). We perform a Gaussian sampling of quasimomenta with standard deviation $\sim 0.1 k_{\mathrm{L}}$, in rough accordance with the measured BEC temperature of around 10-15 nK. For simulation of the stochastic kicking protocol, we use the same techniques and additionally average over 100 different realizations of the fluctuations (note this is slightly different than in the experiment where a single kick period disorder realization is used). 


\section{Supplementary Files}

This is a list of supplementary files associated with this preprint. Click to download.

- QKRsuppinfoNatphys.pdf 